\title{
Acute Arteriobiliary Fistula Emergent Long after Biliary Drainage and Radioembolization
}

\author{
Murat Dökdök ${ }^{1} \quad$ Kutlay Karaman ${ }^{1}$ \\ ${ }^{1}$ Radiology Department, Anadolu Medical Center Hospital Affiliated \\ with John's Hopkins, Gebze-Kocaeli, Turkey \\ J Clin Interv Radiol ISVIR 2022;6:205-206.
}

\author{
Address for correspondence Murat Dokdok, MD, Anadolu Medical \\ Center Hospital Affiliated with John's Hopkins, Cumhuriyet M. 2255, \\ S. No 3, Gebze 41400, Kocaeli, Turkey \\ (e-mail: Murat.dokdok@anadolusaglik.org).
}

\begin{abstract}
Keywords

- late-onset hemobilia

- arteriobiliary fistula

- arterial injury

- radionecrosis

Various causes of hemobilia have been described in the literature, majority being iatrogenic. Other different pathologies such as inflammation and tumors also have been described. A 55-year-old male, who had a history of obstructive jaundice caused by a $4-\mathrm{cm}$ liver mass treated with external-internal biliary drainage and transarterial radioembolization 6 months back, presented with acute hemobilia. Computed tomography demonstrated widespread radionecrosis of the right lobe and extravasation of intravascular contrast from right hepatic artery into common bile duct. Verified on angiography, the arteriobiliary fistula was managed with coil embolization.
\end{abstract}

\section{Introduction}

Although rare, a variety of conditions, including percutaneous biliary procedures and liver tumors, may lead to hemobilia. ${ }^{1,2}$ Late presentation is merely described in a couple of manuscripts in the literature. ${ }^{3}$ We present an acute arteriobiliary fistula that emerged 6 months after biliary drainage and subsequent transarterial radioembolization (TARE) in a patient with cholangiocarcinoma.

\section{Case Report}

A 55-year-old male who presented with obstructive jaundice caused by a magnetic resonance imaging (MRI)-proven 4-cm liver mass in segment six was referred for biliary drainage. The tumor had invaded the right portal vein and the main bile duct while abutting the right hepatic artery as revealed in the MRI. An external-internal biliary drainage catheter was placed, and biopsy was proven to be a cholangiocarcinoma. Our institutional multidisciplinary tumor board recommended TARE and concomitant systemic chemotherapy. The initial planning arteriography demonstrated tumor vas- cularity supplied from the right hepatic artery. Right lobar TARE with $7 \mathrm{GBq}$ of yttrium-90 glass microspheres was performed to deliver an estimated dose of $110 \mathrm{~Gy}$ to the treatment volume and $190 \mathrm{~Gy}$ to the tumor volume. After four cycles of systemic chemotherapy including gemcitabine and cisplatin, surveillance MRI was performed 3 months posttreatment which showed necrosis and size reduction of the tumor from the maximal width of 38 to $26 \mathrm{~mm}$ indicating partial response according to RECIST 1.1.

Clinical findings and pathological investigations had been unremarkable during follow-up except for somewhat fluctuating liver functions. Six months after TARE, the patient was brought to the emergency department with hematemesis and collapse. Heart rate was slightly high at 116 beats per minute, blood pressure was around $90 / 55 \mathrm{~mm} \mathrm{Hg}$, and hemoglobin level was $8.2 \mathrm{~g} / \mathrm{dL}$. Patient had deteriorated when upper gastrointestinal endoscopy was scheduled. An emergency computed tomography depicted widespread necrosis of the right lobe and extravasation of intravascular contrast agent into common bile duct ( - Fig. 1A, B). This finding was confirmed by a catheter angiography, which showed a right hepatic artery fistula to common bile duct. The segmental branches of the right hepatic article published online February 17, 2022
DOI https://doi.org/ 10.1055/s-0041-1740569. ISSN 2457-0214. (c) 2022. Indian Society of Vascular and Interventional Radiology. All rights reserved.

This is an open access article published by Thieme under the terms of the Creative Commons Attribution-NonDerivative-NonCommercial-License, permitting copying and reproduction so long as the original work is given appropriate credit. Contents may not be used for commercial purposes, or adapted, remixed, transformed or built upon. (https://creativecommons.org/ licenses/by-nc-nd/4.0/)

Thieme Medical and Scientific Publishers Pvt. Ltd., A-12, 2nd Floor, Sector 2, Noida-201301 UP, India 

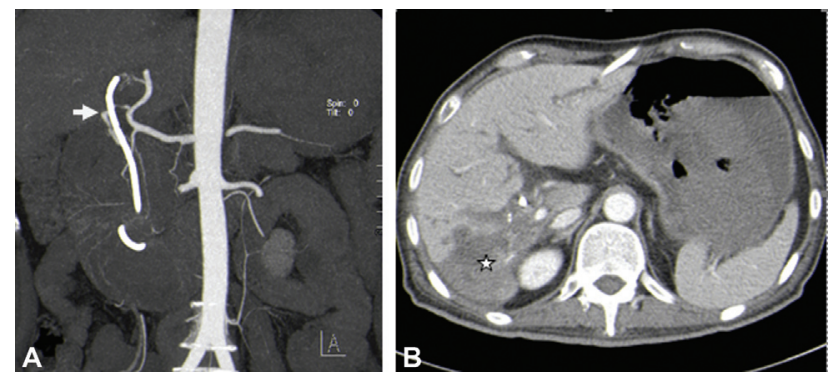

Fig. 1 Computed tomography (CT) angiography shows extravasation of contrast agent into the common bile duct (white arrow) which is seen coursing along the drainage catheter (A). Widespread necrosis of the right lobe (white asterisk) is noted on portal phase contrastenhanced CT (B).

artery distal to the fistula were not filling on angiography. The proximal right hepatic artery displayed diffuse wall irregularity starting from its origin up to the fistula site which was new when compared with the angiography performed before TARE. The fistula was catheterized with a $2.7 \mathrm{~F}$ microcatheter (Progreat; Terumo, Tokyo, Japan) placed coaxially through a $5 \mathrm{~F}$ Simmons- 1 catheter (Radiofocus Glidecath; Terumo). The entire segment of the proximal right hepatic artery was embolized with two 0.018-inch pushable hydro-coils (Azur; Terumo) ( - Fig. 2A, B). The patient was stable after the procedure and did not need any further transfusions.

\section{Discussion}

A fistula between the biliary tree and the accompanying vascular structures-either the hepatic arterial or portal venous system-is a rare cause of upper gastrointestinal bleeding. Percutaneous biliary intervention is the most common cause of this condition, while other causes such as inflammation and liver tumors are infrequent. ${ }^{1}$ To the best of our knowledge, there is only one reported case of arteriobiliary fistula related to chemoradiotherapy of intrahepatic cholangiocarcinoma. ${ }^{2}$ Hemobilia might rarely be observed months or even years after the procedure. ${ }^{3}$ Since an arterial injury is the most common cause in such cases, it requires immediate management due to severity of the clinical presentation. ${ }^{3}$

TARE is utilized increasingly in intrahepatic cholangiocarcinomas as the efficacy of systemic chemotherapy is limited. While biliary obstruction does not pose an absolute contraindication, radioembolization is not proposed in hilar cholangiocarcinomas. Biliary complications including radiation cholecystitis, radiation-induced cholangitis, abscess, bilomas, strictures, and necrosis have been reported up to $10 \%$ after TARE. Microvascular injury and vasculitis of biliary plexus were the proposed biliary injury mechanism in these cases. ${ }^{4}$ Widespread necrosis is accepted to be the principal culprit in major arterial injuries seen after radiotherapy of head and neck tumors. At the same time, radiation has also been implicated to weaken the arterial wall by obliterating the vasa vasorum. ${ }^{5}$

In our case, an external-internal drainage catheter had been placed through the intrahepatic cholangiocarcinoma before TARE. During the 6-month follow-up, continuous

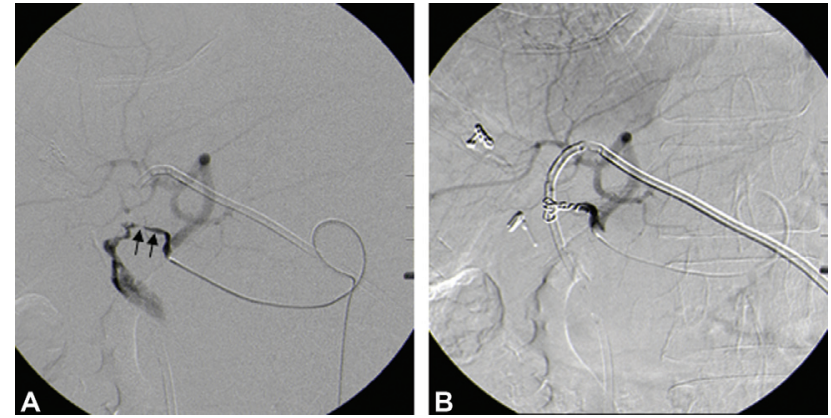

Fig. 2 Proximal right hepatic artery (arrows) demonstrates wall irregularity starting from its origin up to the fistula site $(A)$.

Postembolization angiogram showing coil embolized proximal right hepatic artery with pushable hydro-coils (B).

erosion due to drainage catheter and developing radiation necrosis of tumor in the vicinity of hepatic hilum could have led to hepatic artery injury and arteriobiliary fistula. Unlike other direct hepatic artery injuries that usually spare vascular structures other than the fistula site, we observed wall irregularity throughout the right hepatic artery. Therefore, coil embolization of the entire parent artery starting from its origin up to the biliary fistula site was performed. Hayano et al proposed that presence of hepatic arterial wall irregularity in the segment proximal to the fistula may suggest tumoricidal effects of radiotherapy extending beyond the tumor to involve the blood vessels in the radiated depth as the possible cause for hepatic artery injury rather than direct catheter-induced erosion. ${ }^{2}$ This finding was similar to ours in which the right hepatic artery showed wall irregularity in its entire proximal segment up to the fistula compared with a normal angiogram 6 months prior. Given the imaging findings, we think both factors-tumor necrosis and catheter erosion-might have played a role in our case.

\section{Conclusion}

TARE of liver tumors that cause biliary obstruction could cause unpredictable large necrosis; such radiation-induced necrosis if affecting an encased blood vessel may contribute to late presenting arterial injuries.

\section{Conflict of Interest}

None declared.

\section{References}

1 Green MH, Duell RM, Johnson CD, Jamieson NV. Haemobilia. Br J Surg 2001;88(06):773-786

2 Hayano K, Miura F, Amano H, et al. Arterio-biliary fistula as rare complication of chemoradiation therapy for intrahepatic cholangiocarcinoma. World J Radiol 2010;2(09):374-376

3 Born P, Rösch T, Sandschin W, Weiss W. Arterial bleeding as an unusual late complication of percutaneous transhepatic biliary drainage. Endoscopy 2003;35(11):978-979

4 Riaz A, Awais R, Salem R. Side effects of yttrium-90 radioembolization. Front Oncol 2014;4:198

5 Cohen J, Rad I. Contemporary management of carotid blowout. Curr Opin Otolaryngol Head Neck Surg 2004;12(02):110-115 